\title{
Coulisses
}

Revue de théâtre

42 | Printemps 2011

Racine : Théâtre et émotion

\section{Andromaque et Pyrrhus : le conflit des images et des « faces »}

Sabine Gruffat

\section{(2) OpenEdition}

12 Journals

Édition électronique

URL : https://journals.openedition.org/coulisses/607

DOI : 10.4000/coulisses.607

ISSN : 2546-9460

Éditeur

Presses universitaires de Franche-Comté

Édition imprimée

Date de publication : 15 février 2011

Pagination : 43-54

ISBN : 978-2-84867-316-5

ISSN : 1150-594X

\section{Référence électronique}

Sabine Gruffat, "Andromaque et Pyrrhus : le conflit des images et des « faces » , Coulisses [En ligne], 42 | Printemps 2011, mis en ligne le 30 novembre 2016, consulté le 29 décembre 2022. URL : http:// journals.openedition.org/coulisses/607 ; DOI : https://doi.org/10.4000/coulisses.607 


\title{
Andromaque et Pyrrhus : le conflit des images et des « faces »
}

\author{
SABINE GRUFFAT \\ Université Jean Moulin - Lyon 3
}

L'importance de l'image dans les tragédies raciniennes n'est plus à démontrer et est même devenue un lieu commun de la critique : on parle avec Jean Starobinski de " poétique du regard $»^{1}$ pour évoquer la souffrance sourde de ces êtres hantés par la vision de l'objet désiré et toujours inaccessible ; on évoque dans la lignée de Roland Barthes le «tenebroso racinien » qui, dans le dédoublement des images contrastées, condense les déchirures d'un éros torturé, la dialectique du bourreau et de sa victime. On se plait enfin à ces hypotyposes hallucinatoires qui projettent sur scène des images mentales, des explosions émotionnelles, prêtent un corps aux fantômes d'Oreste ou aux rêves avortés de Phèdre. On le sait donc, la tragédie racinienne se lit dans les regards et se déchaîne par le truchement d'images : images de soi que l'on maquille pour séduire ou impressionner l'autre ; images de l'autre qui réactivent les fantasmes de scènes primitives ; images conflictuelles tendues vers leur improbable résolution.

Andromaque, qui constitue sans doute avant tout une tragédie de la culpabilité et de la mémoire, est traversée par ces pensées obsédantes. Le souvenir de Troie fonctionne comme une anamnèse figeant les deux protagonistes dans des rôles intenables: les cendres de la ville hantent Pyrrhus comme une vision cauchemardesque s'interposant entre lui et la femme aimée, entre lui et une image aimable. Or la veuve qui se nourrit du passé pour commémorer les morts lui interdit cette amnésie salutaire en se faisant l'image vivante du deuil. Ainsi, tous deux portent en eux l'« épaisse nuit» du carnage dont l'évocation empoisonne chacune de leur rencontre et fixe leur destin. On aimerait restreindre le champ de cette vaste question aux relations unissant l'ethos et le

1. Jean Starobinski, L'CEil vivant, Gallimard, 1999.

2. Roland Barthes, Sur Racine, Éditions du seuil, 1963, p. 26-28. 
pathos - autrement dit la production de l'image et sa réception - et, en empruntant à la théorie des «faces » de la pragmatique, examiner en quoi l'image est le lieu d'un travail fragile de négociation entre des aspirations contradictoires. On s'appuiera en particulier sur deux passages de la pièce, l'acte I, scène 4 et l'acte II, scène 5, où la veuve d'Hector et le fils d'Achille sont aux prises avec les valeurs et les désirs inacceptables de l'autre.

Avant d'aborder la façon dont les deux personnages tissent une relation complexe au sein de leurs échanges, il convient sans doute de rappeler que ce conflit des images engage d'abord l'horizon d'attente du public classique et les intentions du dramaturge. Dans ses deux préfaces de 1668 et 1676, Racine met en effet en lumière les modifications qu'il a apportées à ses sources antiques afin d'accommoder son sujet à la culture de ses contemporains. Il en appelle d'abord à leurs connaissances pour faire valoir sa fidélité aux Anciens et signaler ensuite des ajustements qu'il commente aussitôt :

Mais véritablement mes personnages sont si fameux dans l'Antiquité, que, pour peu qu'on la connaisse, on verra fort bien que je les ai rendus tels que les anciens poètes nous les ont donnés. Aussi n’ai-je pas pensé qu'il me fût permis de rien changer à leurs mœurs. Toute la liberté que j’ai prise, ç’a été d'adoucir un peu la férocité de Pyrrhus, que Sénèque, dans sa Troade, et Virgile, dans le second de l'Énéide, ont poussée beaucoup plus loin de que je n'ai cru le devoir faire.

Encore s'est-il trouvé des gens qui se sont plaints qu'il s'emportât contre Andromaque et qu'il voulut épouser une captive à quelque prix que ce fût. J'avoue qu'il n'est pas assez résigné à la volonté de sa maittresse, et que Céladon a mieux connu que lui le parfait amour. Mais que faire ? Pyrrhus n'avait pas lu nos romans. Il était violent de son naturel. Et tous les héros ne sont pas faits pour être des Céladons ${ }^{3}$.

La tragédie, dédicacée à Henriette d'Angleterre, « l'arbitre de tout ce qui se fait d'agréable » à la cour, doit répondre aux attentes du public mondain en adaptant la situation violente des personnages grecs à la galanterie de l'âge classique. La « liberté » que s'accorde l'auteur tâche précisément de concilier le caractère cruel du personnage antique, meurtrier impitoyable de Priam et de Polyxène, avec les bienséances et la civilité modernes. Mais cette concession faite aux conventions de la politesse ne doit pas pour autant contribuer à efféminer le héros ${ }^{4}$, à le rendre ridicule et invraisemblable. C'est bien la nécessité d'un équilibre, d'une conciliation acceptable que pointe l'allusion ironique aux fervents

3. Racine, Première préface d'Andromaque.

4. Si Subligny accusait le Pyrrhus de Racine de n'avoir plus rien de commun avec le caractère violent et farouche que lui prête la tradition, le Grand Condé qui appartenait au cercle de Madame, estimait que le roi montrait encore trop de barbarie envers sa captive. 
partisans de l'Astrée. Racine doit donc «adoucir» l'image sanglante et barbare de Pyrrhus que les lecteurs de Virgile ou Sénèque ont en mémoire pour répondre aux normes esthétiques et morales de ses spectateurs. S'il se heurte là à l'un des problèmes majeurs qui se pose aux dramaturges du XVII ${ }^{e}$ siècle, tenus d'articuler la douceur inhérente à l'honnêteté galante à la fermeté du guerrier viril, il entend fonder sa poétique tant sur le respect des mœurs policées que sur celui des préceptes d'Aristote faisant du héros médiocre le principe même de la bonne tragédie.

Dans la seconde préface, il s'intéresse davantage au personnage d'Andromaque et réfute, toujours au nom de l'idée que le public contemporain se fait de cette héroïne, l'existence d'un fils né de son union avec Pyrrhus :

Andromaque ne connait point d'autre mari qu'Hector, ni d'autre fils qu'Astyanax. J'ai cru en cela me conformer à l'idée que nous avons maintenant de cette princesse. La plupart de ceux qui ont entendu parler d'Andromaque ne la connaissaient guère que pour la veuve d'Hector et pour la mère d'Astyanax. On ne croit point qu'elle doive aimer ni un autre mari, ni un autre fils. Et je doute que les larmes d'Andromaque eussent fait sur l'esprit de mes spectateurs l'impression qu'elles y ont faite, si elles avaient coulé pour un autre fils que celui qu'elle avait d'Hector's.

Racine en appelle à l'imaginaire collectif qui considère Andromaque comme le symbole de la fidélité, de l'exclusivité : elle est la veuve d'un homme et la mère d'un enfant. La préface souligne que cette dignité correspond aux attentes des spectateurs mondains appréciant tout autant les situations pathétiques que la noblesse de caractère ${ }^{6}$. Mais le dramaturge mêle sans doute aux goûts de ses contemporains ses propres impressions de lecture. On a remarqué en effet que l'élève de Port Royal avait annoté son exemplaire de l'Iliade, privilégiant le chant VI et le couple exemplaire que forment Andromaque et Hector ${ }^{7}$. Il admirait l'émotion de cette dernière rencontre, l'angoisse des époux craignant leur sort respectif, le dévouement de la troyenne qui «n'aime que lui ». Il fut également marqué par le livre III de l'Énéide au point de reprendre les mêmes vers dans ses deux préfaces. Longeant les côtes de l'Épire, des années après la guerre de Troie, le héros aperçoit Andromaque recueillie sur le cénotaphe d'Hector et déplorant son passé misérable : elle se fait le porte-parole de toutes les captives arrachées à leur patrie, livrées à leurs vainqueurs, forcées d'« enfante[r] dans la servitude » et rappelle, qu'après avoir dû supporter l'insolence de Pyrrhus, elle a été abandonnée au profit d'Hermione et offerte à Hélénus, son beau-frère réduit également en esclavage. Donnée à d'autres hommes, Andromaque est

5. C'est nous qui soulignons.

6. La tragédie remporta un grand succès lors de sa représentation. Mme de Sévigné, qui vit la pièce en Bretagne, explique que ce personnage de mère douloureuse lui « fit pleurer plus de six larmes ». (Lettre du 12 août 1671).

7. Susanna Philippo, "The Legacy of Homer: The Iliad Annotations of Jean Racine", L'Antiquité classique, vol. 65, 1996, p. 1-29. 
néanmoins présentée comme une veuve ensevelie dans le souvenir de son époux, comme un monument vivant érigé à la mémoire de toutes les souffrances causées par la guerre. On comprend dès lors que Racine ait cherché à épurer cette figure en ne retenant d'elle que le fond de son caractère, en lui épargnant en quelque sorte des vicissitudes accidentelles. C'est pourquoi il prétend se démarquer tout à fait de l'héroïne d'Euripide qui « craint pour la vie de Molossus », fils de Pyrrhus, qu'Hermione jalouse cherche à faire périr.

La tragédie grecque s'ouvre sur le monologue de l'ancienne reine qui, après avoir pleuré son passé troyen, rappelé le sort funeste d'Hector et d'Astyanax, avoue considérer son dernier fils comme un appui et une consolation. Elle apparaît alors folle d'inquiétude à l'idée qu'Hermione, en l'absence de Pyrrhus, s'en prenne à son « unique espérance ». Davantage, lorsque sa rivale, pleine de mauvaise foi, lui reproche ses mœurs dénaturées et l'accuse d'avoir eu des enfants d'un meurtrier, elle lui explique qu'une bonne épouse, non seulement cherche à ne pas déplaire à son époux, mais doit encore accueillir ses éventuels bâtards ${ }^{8}$. Enfin, soumise à un chantage par Ménélas, Andromaque est prête à sacrifier sa vie afin de sauver son fils :

Ô mon petit, moi qui t'ai mis au monde, pour t'épargner la mort

je descends vers l'Hadès. Si tu évites l'heure fatale

souviens-toi de ta mère, de ma fin douloureuse $[\ldots]$

Pour tous les hommes je le sais, les enfants sont la vie. (vers 413-415 ; 418-419)

Un tel passage suffit sans doute à montrer que la représentation d'Andromaque reste fondamentalement la même : elle demeure d'une part une figure douloureuse, vouée à revivre sans cesse la perte de son fils, que celui-ci se nomme Astyanax ou Molossus. Sa cohérence tient d'autre part à sa fidélité inaliénable : fidélité à elle-même, à ses sentiments, à ses principes; fidélité qui l'expose à tous les chantages mais qui lui permet aussi de dénoncer depuis sa propre stabilité toutes les déchéances. Si Racine édulcore l'histoire de cette femme ballottée malgré elle d'homme en homme, c'est peut-être autant pour des raisons de bienséances que pour recentrer le personnage sur ce qui fait son essence, sa singularité. Aussi Jean Rohou a-t-il considéré qu'Andromaque et Hector constituent dans la pièce un «axe vertical [qui] exprime l'élan de la déchéance vers la transcendance, [qui] manifeste la quête tragique, par l'être, de son inaccessible raison d'être $»^{9}$. Enlevée à sa famille, exilée, la veuve fonde son identité

8. «Ah mon très cher Hector, pour l'amour de toi j'ai moi-même

Chéri ce que tu chérissais, quand d'aventure t'égarait Cypris,

Et ma propre mamelle, aux petits bâtards nés de toi,

Bien souvent je l'offris, pour que tu n'aies en moi nulle cause d'humeur.

Ainsi par ma vertu je me conciliais mon mari. » Andromaque, Euripide, vers 222-226, Tragédies complètes, t. I, trad. par Marie Delcourt-Curvers, Gallimard, 1962, p. 352.

9. Jean Rohou, L'évolution du tragique racinien, Paris, SEDES, 1991, p. 64. 
sur le culte de ses valeurs, ancre son être dans l'attachement à sa foi morale et à son devoir.

Les modifications apportées aux sources antiques témoignent donc à la fois d'une appropriation personnelle du mythe et d'une interprétation de la tragédie: si Racine conserve les principaux protagonistes, c'est pour les replacer dans des perspectives et des rapports de force différents. En préservant à tout prix la chasteté d'Andromaque, le dramaturge privilégie la pureté morale de son héroïne et l'idéalise comme veuve et comme mère. Le personnage domine tous les autres de sa grandeur et de sa majesté malgré son statut de captive. En outre, l'intransigeance de l'héroïne permet de suspendre toute l'intrigue à sa décision, de mettre en lumière le péril qui pèse sur la vertu dans un monde dominé par les passions. Ainsi, l'unité d'action apparaît resserrée autour d'une question unique (Andromaque cèdera-t-elle à Pyrrhus ou non pour sauver son fils ?) et elle est étroitement liée aux comportements des personnages dépendant les uns des autres. C'est précisément l'argument dramaturgique que met en avant Jean Rohou pour contrer l'accusation de coquetterie que l'on porta contre la veuve dès le XIX ${ }^{\mathrm{e}}$ siècle, ou plutôt pour déplacer le problème :

La partenaire de Pyrrhus sur une scène parisienne ne saurait être identique à l'idéale veuve d'Hector dans une fiction utopique. Ses paroles et attitudes doivent animer des affrontements de théâtre, où chaque réplique est conçue pour faire réagir le partenaire et repousser ses contre-attaques. C'est l'actrice sinon la provocatrice que le dramaturge doit faire parler pour faire fonctionner ces scènes, c'est-à-dire un personnage radicalement différent de l'image de fidélité que nous présente le moraliste. Leur contradiction ne provient pas de la complexité d'Andromaque et de l'éternel féminin, ni principalement du fait qu'elle soit jetée dans une situation contraire à sa nature, mais de la coexistence sous le même nom d'une fidélité idéale et d'un rôle théâtral ${ }^{10}$.

C'est alors que l'on croise la notion d'ethos que l'on peut articuler à la théorie des «faces » utilisée dans les analyses de l'interaction conversationnelle. On rappellera que :

L'ethos est ce qui donne [...] crédibilité au discours et le pathos, ce qui le fait accepter : les deux moments de la production et de la réception. Est-ce à dire que l'orateur est sans passion et l'auditoire sans vertu ? Les vertus de l'orateur ne sont pas les mêmes, car c'est celui qui agit par le discours, tandis que ses passions doivent se régler sur celles de ceux à qui il s'adresse s'il espère les convaincre ${ }^{11}$.

Autrement dit, l'ethos correspond au rôle que doit savoir interpréter le locuteur pour convaincre ou toucher son interlocuteur, notamment en correspondant à

10. Jean Rohou, Avez-vous lu Racine? Mise au point polémique, Paris, L'Harmattan, 2000, p. 162-163.

11. Michel Meyer dans son introduction à la Rhétorique d'Aristote, Le livre de poche, «Classiques de la philosophie », 1991, p. 34. 
ses attentes. Il désigne une image de soi, construite par un comportement et une façon de parler. Or le sociologue Erving Goffman a proposé un modèle dramaturgique pour étudier les interactions quotidiennes et la manière dont les gens déploient des stratégies afin de négocier leur position face à autrui ${ }^{12}$. Des interlocuteurs qui se plient aux règles de la politesse sont des " figurants » au sens théâtral du terme ${ }^{13}$ qui cherchent chacun à « sauver la face », c'est-à-dire à préserver le territoire de son moi et à valoriser son image auprès d'autrui tout en essayant néanmoins de ménager les faces de son interlocuteur. Mais Goffman s'est aussi intéressé aux cas où la proximité des individus, l'ambiguïté de toute communication compromettent ces règles de politesse et engendrent au contraire un sentiment de menace et d'offense. Il s'est alors attaché à analyser comment s'opérait la gestion des agressions lorsque les principes conversationnels et l'ordre social étaient effectivement perturbés ${ }^{14}$. Nous y reviendrons mais il pourrait être intéressant d'utiliser certains de ces concepts pour aborder une tragédie où un roi amoureux s'efforce de gagner les faveurs de sa captive sans pour autant s'humilier tandis que celle-ci entend sauvegarder l'image sacrée qu'elle a de ses principes sans trop heurter son geôlier. La tension entre les deux personnages est telle que leurs rencontres correspondent systématiquement à des situations remettant en cause l'ordre établi qui garantirait à chacun la protection de sa «face ». On se propose donc d'étudier à présent ces situations de déséquilibre en privilégiant deux extraits.

Lorsqu'il aborde la première entrevue de Pyrrhus et d'Andromaque à la scène 4 de l'acte I, le spectateur sait d'une part que le roi s'adonne à un véritable chantage affectif sur sa captive, et d'autre part qu'il s'oppose à tous les grecs en prenant la défense d'Astyanax. Le tête-à-tête est donc placé sous un éclairage ambigu : Pyrrhus commence par susciter les angoisses de la mère en lui parlant de la mission d'Oreste pour lui offrir ensuite son appui en gage d'amour :

Je vous offre mon bras. Puis-je espérer encore

Que vous accepterez un cœur qui vous adore?

En combattant pour vous, me sera-t-il permis

De ne vous point compter parmi mes ennemis ? (vers 293-296)

En tendant une image chevaleresque de lui-même, le roi se réclame d'un ethos héroïque et vertueux, d'une noblesse de caractère qui est censée rassurer Andromaque et lui attirer peut-être sa reconnaissance. Pour gagner sa confiance,

12. Voir en particulier La Présentation de soi, Éditions de Minuit, coll. «Le Sens Commun », 1973. L'auteur recourt notamment aux métaphores de la «scène », du "personnage », du "rôle », des «coulisses » pour évoquer les attitudes explicites et surtout implicites qui entrent dans toute relation sociale.

13. On reprend l'expression de Catherine Kerbrat-Orecchioni, Le Discours en interaction, Armand Colin, 2005, p. 194.

14. E. Goffman, Les Rites d'interaction. Paris, Minuit, 1974. 
il adhère aux valeurs morales de son interlocutrice : c'est ce qu'Aristote appelle la "preuve éthique » qui permet à l'orateur de se montrer sous un jour favorable. Mais les deux interrogatives, en sollicitant une reconnaissance, tendent à substituer au désintéressement attendu une logique de la rétribution, du marchandage. Le vocabulaire amoureux croise le lexique guerrier pour maintenir une équivoque. Dès lors on peut voir dans cet ethos galant et héroïque une tentative de reconstruction de soi (l'ethos assignerait en quelque sorte un devoir être, commanderait une ligne d'action). Dans cette perspective, Pyrrhus poserait les fondements d'un avenir prometteur, faisant table rase du passé. On peut y reconnaitre aussi une ruse argumentative, une manœuvre manipulatrice : l'ethos renverrait bien alors à une identité fictive visant avant tout à capter les sentiments de l'interlocutrice. Sa stratégie vise à ménager un équilibre entre les faces sans pour autant réduire tout à fait toute menace : en faisant sa requête, Pyrrhus s'expose à une rebuffade mais laisse entendre en même temps le pouvoir et le statut qui sont les siens. Or Andromaque choisit de gommer l'ambiguité du discours pour attirer son adversaire sur le terrain du devoir et de la morale :

Seigneur, que faites-vous, et que dira la Grèce?

Faut-il qu'un si grand cœur montre tant de faiblesse?

Voulez-vous qu'un dessein si beau, si généreux,

Passe pour le transport d'un esprit amoureux ?

Captive, toujours triste, importune à moi-même,

Pouvez-vous souhaiter qu'Andromaque vous aime?

Quels charmes ont pour vous des yeux infortunés

Qu'à des pleurs éternels vous avez condamnés ?

Non, non, d'un ennemi respecter la misère,

Sauver des malheureux, rendre un fils à sa mère,

De cent peuples pour lui combattre la rigueur

Sans me faire payer son salut de mon cœur,

Malgré moi, s'il le faut, lui donner un asile :

Seigneur, voilà des soins dignes du fils d'Achille. (vers 297-310)

Andromaque commence par relever la contradiction qui anime Pyrrhus et oppose l'ethos discursif - l'image de l'orateur au sein de son discours - et l'être véritable, le discours et ses implications. Elle prend soin cependant de ne pas opposer d'abord son désir à celui de Pyrrhus mais l'éthique dont se réclame Pyrrhus et ce qu'elle suppose. Ce détour lui permet de ne pas froisser le roi tout en essayant de susciter chez lui des sentiments susceptibles de contrebalancer sa passion. En dosant reproches et flatteries, elle s'efforce en effet de l'attirer sur le terrain des passions nobles que sont la générosité, le courage et la gloire et de le détourner de la concupiscence amoureuse. Aussi le conflit est-il déplacé : au couple problématique que constituent Pyrrhus et Andromaque, la captive substitue un autre couple en rappelant au roi ses devoirs envers la Grèce. En inscrivant son interlocuteur dans une logique strictement politique, 
elle lui restitue par là même un caractère "sacré », au sens où l'entend Ervin Goffman : « la face est un objet sacré, et il s'en suit que l'ordre expressif nécessaire à sa préservation est un ordre rituel $»^{15}$. Et cet ordre rituel dessine une contrainte sociale fondamentale à laquelle les deux protagonistes doivent se plier. C'est pourquoi Andromaque se pose en veuve troyenne et parle d'elle à la troisième personne : loin de voir en Pyrrhus un époux potentiel, elle lui rappelle le passé, leur identité nationale qui ont figé à jamais le reste de sa vie et leurs rôles respectifs. Ayant signifié l'impossibilité, l'inconvenance, voire la monstruosité d'un tel amour, elle invite son interlocuteur à remplir l'image idéale qu'il a esquissée de lui. Elle oppose à son désir la magnanimité d'un vrai héros. Ce deuxième portrait qu'elle brosse de lui constitue une sorte d'image en creux que le roi doit venir remplir. Mais l'on quitte alors la réalité politique pour entrer dans une fiction utopique qui fait pendant à l'image invraisemblable d'un couple réconcilié. C'est une conversion à la charité, pour ne pas dire une rédemption que mime le mouvement de cette longue phrase qui enchaîne des infinitifs à valeur prescriptive. On notera au passage qu'en utilisant le vocabulaire de la rétribution ("me faire payer»), Andromaque dévoile au grand jour le chantage que suggérait le discours de Pyrrhus; mais elle adoucit en même temps cette agression en exaltant la mission héroïque du roi. Ce double jeu fragile, qui suscitera l'agacement de Pyrrhus, met en lumière ce conflit des faces toujours susceptible de déraper.

Andromaque parvient donc dans cet extrait à renverser la situation en pratiquant une rétorsion: s'appuyant sur l'ethos que revendique Pyrrhus pour la séduire, elle retourne contre lui cette image chevaleresque qui implique des devoirs moraux. Elle fait alors glisser son interlocuteur d'un ethos qui n'était qu'une stratégie argumentative, une image discursive ou une construction utopique, à un impératif éthique. C'est au nom de la générosité et de la gloire qu'elle demande à son bourreau d'oublier une passion coupable et inadmissible et de sauver son enfant sans contrepartie. Cette opposition des passions nobles à la concupiscence restera sans effet sur Pyrrhus qui, à la fin de la scène, explicite son chantage. Ce premier face-à-face montre néanmoins que la notion d'ethos est au cœur de la tragédie : elle peut permettre de révéler la ruse du tyran éconduit ou dévoiler la tragédie d'un caractère, le dilemme du personnage pris entre ce qu'il a été et ce qu'il aspire à être; elle signale l'abyme qui sépare deux systèmes de valeurs - Andromaque trouve son devoir être dans son veuvage - ; elle traduit enfin la volonté de Racine d'insuffler une nouvelle complexité à ses personnages en ménageant du jeu dans leur image théâtrale.

La scène 5 de l'acte II est à lire comme la réponse d'Andromaque aux conseils menaçants du roi qui l'invitait à se ressaisir en retrouvant son fils. La

15. Ibid., p. 21. 
conversation de Pyrrhus avec son confident nous apprend que la veuve l'a humilié et qu'il entend désormais se détourner d'elle. Il entreprend néanmoins de retracer à Phoenix cette entrevue à laquelle ce dernier a assisté mais qui a eu lieu dans les coulisses. Au-delà de sa valeur informative, ce récit est dès lors l'occasion pour Racine d'articuler diverses mises en scène, de donner toute son ampleur au conflit des images dans un contexte de transgression apparente de l'ordre établi. Tout l'extrait, saturé par le lexique de la vision, nous conduit en effet d'image en image : l'image espérée et fantasmatique d'Andromaque suppliante ; l'image vue et bien réelle d'Andromaque révoltée ; l'image vengeresse d'Andromaque à genoux. On va donc essayer de montrer comment ces quelques vers supposent clairement que chaque personnage cherche à manipuler l'autre par le biais de l'image, à le faire entrer dans sa mise en scène.

Tu l'as vu, comme elle m'a traité.

Je pensais en voyant sa tendresse alarmée,

Que son fils me la dût renvoyer désarmée.

J'allais voir le succès de ses embrassements :

Je n'ai trouvé que pleurs mêlés d'emportements.

Cent fois le nom d'Hector est sorti de sa bouche.

Vainement à son fils j'assurais mon secours :

«C'est Hector, disait-elle, en l'embrassant toujours ;

Voilà ses yeux, sa bouche, et déjà son audace ;

C'est lui-même, c'est toi, cher époux, que j'embrasse. »

Et quelle est sa pensée? Attend-elle en ce jour

Que je lui laisse un fils pour nourrir son amour? (vers 644-656)

L'acte de voir, la mise en scène de l'autre est d'emblée présentée comme une humiliation, comme un acte menaçant la face du roi-spectateur. Et l'offense apparaît d'autant plus grande qu'elle a eu lieu en public, comme le rappelle la prise à parti de Phoenix. Goffman distingue à ce propos l'insulte légère ou involontaire qui peut être évacuée par une stratégie d'évitement. Si l'outrage semble au contraire délibéré, le dérèglement de l'ordre rituel est perçu comme une véritable profanation de la face de l'interlocuteur et appelle une réparation. Tout à sa colère et à sa stupéfaction, Pyrrhus rejoue la scène vexatoire dans le cadre du dialogue et de la remémoration. Il commence par évoquer les espoirs qu'il avait fondés sur les retrouvailles de la mère et du fils. En même temps, la construction subjective qu'explicite le verbe de pensée et la modalisation («je pensais [...] Que son fils me la dût renvoyer») signalent au spectateur la cruauté blessante que le roi exerçait sur ses prisonniers. Le renversement brutal de situation souligne alors à la fois l'amertume de Pyrrhus trompé dans ses attentes et la réplique d'Andromaque répondant à l'agression par un comportement vexatoire. Le décrochage du discours direct nous donne alors à voir la mise en scène intolérable à laquelle le roi impuissant a dû assister, relégué au rang de spectateur extérieur mais bien présent. L'identification du fils au mari a 
déjà fait couler beaucoup d'encre, notamment dans le but de signifier à quel point Andromaque déterminait sa conduite par rapport à son mari et se comportait davantage en amante qu'en mère. Il est inutile de revenir ici sur ce débat. On retiendra surtout la mise en scène du corps de l'être aimé, désigné et exposé dans ses diverses parties, comme pour reconstruire le territoire d'Andromaque, ce territoire qu'a bafoué Pyrrhus et auquel elle redonne une existence charnelle et spatiale. La scène - l'espace théâtral -, que le récit met en abyme délimite en effet un lieu intime, conjugal et sacré ${ }^{16}$ et les embrassements, au-delà de leurs connotations érotiques, participent avant tout d'un cérémonial visant à rappeler, à exhiber l'identité fondamentale de la troyenne. Se superposent dès lors deux réactions, deux réceptions : Pyrrhus, replié sur son amourpropre offensé et plein de son pouvoir, perçoit l'offense comme une aberration (" et quelle est sa pensée ? »), comme un signe de folie puisque la transgression de l'autorité, la profanation des faces ne peuvent que mettre en danger Andromaque et son fils. Le spectateur de la tragédie, qui n'est pas asservi à cette passion dévorante ni happé par cette mise en scène sidérante, est à même de reconstituer la logique des événements et de restituer à cette vision sa portée symbolique. Comme l'a écrit Jean Starobinski, «ce qu'attendait le personnage racinien, c'était le regard caressant, la douce prise amoureuse ; ce qu'il découvre en réalité, c'est sa propre culpabilité [car] dans tout regard désirant, il y a d'avance une transgression, le viol d'un interdit, le commencement d'un crime $»^{17}$. C'est cette transgression que dénonce brutalement le théâtre d'Andromaque : son jeu de rôle signale à Pyrrhus qu'il a bafoué des sentiments sacrés, un ordre rituel que représente l'étreinte.

C'est par une autre image, par une redistribution des rôles que l'amant éconduit entend se venger :

Je vois ce qui la flatte :

Sa beauté la rassure ; et, malgré mon courroux,

L'orgueilleuse m'attend encore à ses genoux.

Je la verrais aux miens, Phoenix, d'un œil tranquille.

Elle est veuve d'Hector, et je suis fils d'Achille :

Trop de haine sépare Andromaque et Pyrrhus. (vers 658-663)

La scène fantasmée trouve son point focal dans l'amour-propre du roi malmené. L'œil du prince organise le spectacle selon des critères erronés qui substitue à l'interaction sociale un rituel sadique et égocentrique. La première occur-

16. On renverra à ce propos à la mise en scène qu'avait proposée Declan Donnellan au Théâtre des Bouffes du Nord en 2007 : ayant choisi de faire figurer Astyanax sur le plateau, il avait montré l'îlot que composaient, le territoire que délimitaient sur scène la mère et le fils, l'amante et son époux sous les yeux de Pyrrhus situé à quelques pas seulement. Il s'agissait toujours d'une représentation mentale mais à laquelle la mise en scène donnait toute sa présence.

17. Jean Starobinski, L'ail vivant, op. cit., p. 90. 
rence du verbe « voir » confirme l'incompréhension profonde des motivations de l'offense : Pyrrhus imagine Andromaque misant sur ses charmes pour renverser les rapports de force et soumettre le roi à sa volonté. Aux prétendues illusions de la veuve, le grec oppose son propre tableau chimérique. La réponse à l'humiliation réside dans une surenchère de la cruauté : Pyrrhus s'imagine en voyeur jouissant de la souffrance et de la mortification de l'autre. Ainsi, la provocation appelle une autre provocation; la réparation dans l'univers tragique est inenvisageable et semble devoir disparaitre devant le cycle infernal de la violence. Mais cette ultime mise en scène qui oppose le bourreau à sa victime, le roi à sa captive se réclame en fait d'une distribution des rôles qui dépasse les volontés individuelles. Andromaque et Pyrrhus sont pris dans l'Histoire qui leur a fixé des rôles d'ennemis. Et c'est bien à cet ordre supérieur que fait allusion Pyrrhus en s'inscrivant dans une logique nationale pour asseoir sa décision, pour opposer les exigences de la politique à la passion. Renouant momentanément avec le raisonnement d'Andromaque, le «fils d'Achille» se raccroche aux intérêts transcendants de la Grèce, aux contraintes de la patrie et aux lois de la guerre. Mais c'est sans doute parce que cet argument lui paraît trop artificiel et fallacieux, parce qu'il risque de conduire Pyrrhus à une autohumiliation que Phœnix s'efforce de mettre un terme à ce discours, à cette illusion de vengeance ${ }^{18}$. Pour le spectateur averti et distancié, l'échange aura mis en lumière la façon dont les personnages raciniens agissent les uns sur les autres par le biais de l'image théâtrale et sur le mode de la violence : il s'agit toujours d'imposer à l'autre un spectacle indésirable, un rôle finalement humiliant. On connaît la suite de la pièce : l'urgence de la situation, le péril pesant sur son fils contraindra Andromaque à rassurer Pyrrhus, à épargner au moins en apparence ses sentiments. Mais son ultime projet - se suicider après le mariage - visera toujours à préserver son intégrité en sortant définitivement de scène. Ses derniers conseils à Astyanax consisteront pourtant à préparer son intégration sociale pour qu'advienne peut-être un nouvel équilibre :

Mais qu'il ne songe plus, Céphise, à nous venger :

Nous lui laissons un maittre, il le doit ménager. (vers 1119-1120)

Si Racine s'est donc conformé aux attentes et aux goûts de ses contemporains, c'est en prêtant aux personnages antiques un vocabulaire galant. Mais si le lexique peut rappeler celui des romans précieux, les lois de la conversation diffèrent du tout au tout. Il n'est nullement question sur scène de cette prudence, de cette modération qui permet de «faire son plaisir et celui des autres, [de] ménager leur amour-propre et ne le blesser jamais $»^{19}$, d'éviter toute bassesse et toute injustice au nom d'un idéal social. La tragédie racinienne n’est pas

18. Vers 664 : «Commencez donc, Seigneur, à ne m'en parler plus. »

19. La Rochefoucauld, Réflexions diverses, «De la société », dans Maximes, éd. J. Truchet, Paris, Classiques Garnier, 1999, p. 185. 
le lieu de la politesse mais d'affrontements plus ou moins explicites, de manœuvres cherchant à amener l'autre à servir ses propres intérêts. On a essayé de montrer que la théorie des «faces» pouvait être opératoire pour analyser les stratégies d'interaction d'une tragédie précisément fondée sur une chaîne relationnelle. Les contraintes que chacun fait peser sur l'autre se traduisent en termes de conflits d'images, en calculs égoïstes, en efforts désespérés pour imposer son territoire moral et affectif à l'adversaire. Reste le processus cathartique, fondé sur un jeu d'adhésion et de mise à distance. La confrontation des images et des «faces »- représentation troublée et passionnelle de Pyrrhus, spectacle de la force morale mêlée de désespoir d'Andromaque - tend à révéler aux spectateurs les conséquences d'une interaction échappant aux règles de la sociabilité, d'un « dehors sans repères $»^{20}$.

20. Dominique Maingueneau, Pragmatique pour le discours littéraire, Paris, Dunod, 1997, p. 113. 